\title{
Algunos aspectos limnologicos de un lago altoandino: el lago San Pablo, Ecuador.
}

\author{
Jorge E. Casallas G. y Günter Gunkel
}

Technical University of Berlin, Dept. of Water Quality Control, Sekr. KF 4, Strasse des 17.Juni 135, 10623 Berlin, Germany.jorgfkgi@linux.zrz.tu-berlin.de/gunkel@itu206.ut.tu-berlin.de

\section{RESUMEN}

Entre Enero de 1998 y Diciembre de 1999 se llevaron a cabo estudios limnologicos en el Lago San Pat)lo, Ecuador. Se presentan aqui los resultados obtenidos a partir de los cuales se ha podido establecer el carácter monomictico del lago, con un período de completa circulación durante la epoca de fuertes vientos en los meses de verano (entre Junio y Agosto). Durante el resto del año se presenta una estratificacion termica de baja estabilidad, debida a una diferencia de temperatura de maximo 4.5 "C entre las diferentes capas de agua. Además, debido al descenso de temperatura durante las horas de la noche, se presentan procesos noctumos de mezcla.

La actividad agricola y la erosion en la cuenca del lago, asi como la inexistencia de tratamiento de las aguas residuales, que en parte son vertidas en él, han llevado a un proceso de eutrofizacion. A pesar de esto se ha podido determinar una baja productividad, ya que al parecer el nitrógeno actua como factor limitante. El numero de especies planctonicas es bajo, así mismo su abundancia. El fitoplancton esta representado principalmente por la diatomea filamentosa Aulacoseira granulata, asi como por la Clorofita Scenedesmus linearis y la Euglenofita Trachelomonas volvocina. La comunidad de zooplancton es pobre y esta representada por Daphnia pulicaria, Metacyclops mendocinus, y por los rotiferos Keratella tropica, Trichocercasimilis, Anuraeopsis fissay Asplanchna sp.

Palabras clave: limnología tropical, lagos altoandinos, Ecuador, Lago San Pablo, fitoplancton, zooplancton

\begin{abstract}
The limnology o Lake San Pablo (Ecuador) was followed from January 1998 to December 1999. The monomictic character of the lake was established. A period of full mixing occurred during summer (i.e. between June and August). During the rest of the year the water column was thermally stratified, albeit stratification was of low stability because of small temperature differences down the water column (i.e. max. 4.5 "C). Furthermore, at night convective heat loss takes place, lowering water temperature. Intensive agricultural activities, land erosion at the catchment area and the inflow of untreated wastewater into the lake have contributed to its actual eutrophic state. However; the lake is relatively unproductive, apparently because nitrogen is a limiting nutrient. Few species of both phytoplankton andzooplankton werefound, and densities were low. The phytoplankton was represented primarily by the filamentous diatom Aulacoseira granula, the green algae Scenedesmus linearis and the Euglenophyceae Trachelomonas volvocina. The zooplankton was poor and is represented by Daphnia pulicaria, Metacyclops mendocinus and the rotatoria Keratella tropica, Trichocerca similis, Anuraeopsis fissa and Asplanchna sp.
\end{abstract}

Keywords: tropical limnology, high mountain lakes, Ecuador, Lago San Pablo, phytoplankton, zooplankton

\section{INTRODUCCIÓN}

La mayoria de los trabajos limnologicos realizados hasta el momento han sido llevados a cabo en las zonas templadas. Comparativamente, son pocos los estudios que hasta ahora se han desarrollado en sistemas acuaticos de zonas tropicales, a pesar de la importancia económica (entre otros, como fuente de agua potable y de generación de energia electrica) y sociocultural que dichos sistemas pueden representar en estas regiones. El conocimiento actual sobre los lagos y embalses tropicales se debe en gran parte a los estudios que se han desarrollado en las ultimas decadas, especialmente en zonas bajas del tropico (Lewis, 1973; Nilsen, 1984; Payne, 1986; Melack, 1996). 
Una de las caracteristicas mas sobresalientes de las zonas tropicales es la poca variación que presenta la temperatura a 10 largo del año (List, 1971 en Osborne, 2000), con variaciones determinadas por la altura en metros sobre el nivel del mar (msnm), originando asi pisos termicos con rangos de temperatura estable (Holdridge, 1978). Este hecho a su vez determina que en los lagos de estas zonas las fases de circulación y estratificacion no necesariamente sigan un ritmo anual, tal como ocurre en las zonas templadas, sino que varian de acuerdo a las condiciones locales. En terminos generales, la estratificacion térmica que se presenta en estos sistemas lacustres esta ante todo determinada por las variaciones de temperatura entre el dia y la noche (Loffler, 1964; Ducharme, 1975; Powell et al., 1984; Gunkel \& Casallas, 2001). Ya que de darse una estratificacion esta es poco estable y de corta duración sin formacion de termoclina-, la designación de epilimnio, metalimnio e hipolimnio no es del todo correcta desde el punto de vista termico, siendo mas conveniente definir una zona fotica o trofogenica y otra zona afotica o trofolitica (Roldan, 1992).

Los lagos tropicales de montaña estan loca lizados entre los 2000 y $4000 \mathrm{msnm}$, siendo el lago Titicaca uno de los representantes mas conocidos. Debido a la altitud y a las consecuentes condiciones climaticas imperantes, la dinamica de estos lagos y embalses se aparta considerablemente de la que presentan los sistemas en las zonas bajas del tropico. Investigaciones realizadas en algunos lagos de alta montaña han contribuido al conocimiento del regimen termico de estos, siendo en general clasificados como oli gotérmicos con circulaciones frecuentes (nunca se estratifican), por 10 cual han sido llamados polimicticos fríos (Hutchinson \& Loefler, 1956; Rodán, 1992). Caracteristicas adicionales de estos lagos son el aislamiento geografico, el presentar temperaturas medias por debajo de 20 "C, valores de saturación de oxigeno bajos $(<7 \mathrm{mg} / \mathrm{l})$, y a diferencia de los lagos de zonas bajas, ser por lo general mas profundos.

Los trabajos limnologicos desarrollados hasta ahora en Ecuador son muy pocos y el cono- cimiento que se tiene sobre los sistemas acuaticos es escaso. Steinitz-Kannan et al. (1983) hacen referencia a los primeros trabajos y a los resultados obtenidos en sus investigaciones realizadas a partir de 1966 en algunos lagos ecuatorianos. Miller et al. (1984) presentan los primeros datos de productividad para algunos lagos ecuatorianos, incluido el lago San Pablo, y hacen referencia al impacto de las actividades humanas sobre la productividad en algunos de ellos. Se han realizado en el pais además algunos trabajos taxonomicos relacionados con el fitoplancton (Rott, 1981a), los rotiferos (Koste \& Bottger, 1989, 1992), los ostracodos y copepodos (Loffler, 1963) y sobre el valor bioindicador de las macrofitas en los lagos de montaña (Kiersch et al., 2001). Gunkel (2000) presenta una recopilacion de datos disponibles acerca del lago San Pablo, y Gunkel \& Casallas (200I) hacen una primera evaluación de la limitacion de la productividad causada por mezcla diaria en el lago.

El objetivo principal del presente trabajo ha sido realizar para el lago San Pablo una evalua cion a mediano plazo, con el fin de establecer el estado de las comunidades biologicas y la relacion espacio-temporal de las variables fisicoquimicas que determinan tanto el comportamiento hidrodinamico como el suministro y la distribucion de nutrientes, factores que son determinantes en el establecimiento y desarrollo de las especies que lo habitan.

\section{AREA DE ESTUDIO}

El lago San Pablo esta localizado a $100 \mathrm{~km}$ al norte de la ciudad de Quito, capital del Ecuador, a $\operatorname{los} 0^{\circ} 13^{\prime} \mathrm{N}$ y $78^{\circ} 14^{\prime} \mathrm{W}$. Situado a una altura de $2660 \mathrm{msnm}$, con una superficie de $6.7 \mathrm{~km}^{2}$, profundidad maxima de $35 \mathrm{~m}$, y media de $24.6 \mathrm{~m}$, es el segundo lago mas grande del pais. El lago presenta forma circular, ocupando al parecer una antigua cubeta sobre el piso de una meseta interandina. Las aguas del lago son por lo gene ral tranquilas durante la noche y la mañana, desarrollando fuerte oleaje en las horas de la tarde, especialmente durante la epoca seca 
(Steinitz-Kannan et al., 1983; Gunkel \& Casallas, 2001). La epoca lluviosa esta comprendida entre los meses de Octubre y Mayo y la epoca seca entre Junio y Septiembre (INAMHI). Las temperaturas medias del aire varian entre 8.3 " $\mathrm{C}$ (nocturna) y 22.7 " $\mathrm{C}$ (diurna). La precipitación media anual puede llegar a los $1000 \mathrm{~mm}$ y es algo mayor que la evaporación, la cual llega a $858 \mathrm{~mm}$.

El principal afluente del lago es el rio Itambi, el cual contribuye aproximadamente con el $90 \%$ de los aportes de agua, y el principal efluente es el llamado "Desaguadero"; el tiempo de residencia ha sido estimado en 3.2 afios (Galarraga et al., 1992). La cuenca de captacion del rio Itambi comprende un area montafiosa de $121.33 \mathrm{~km}^{2}$, con areas de cultivos que llegan por encima de los 3400 msnm (Paredes, 1994). La vegetación natural ha desaparecido totalmente, siendo remplazada por cultivos agricolas y algunas areas de bosque secundario, con lo cual se ha intensificado el riesgo de erosion .

En la cuenca del lago esta asentada una poblacion de aproximadamente 20000 habitantes, la cual ejerce una fuerte presion sobre los recursos hidricos. Actualmente el lago San Pablo presenta problemas de eutroficacion debidos a la entrada permanente de aguas residuales con alto contenido de nutrientes provenientes de la agricultura intensiva, de los asentamiento humanos presentes en su cuenca y de una incipiente infraestructura turistica asentada en sus orillas.

\section{MATERIALES Y MÉTODOS}

\section{Parametros fisico-quimicos}

Entre Enero de 1998 y Diciembre de 1999 se llevaron a cabo muestreos quincenales (1998) y mensuales (1999) en la zona mas profunda del lago $\left(00^{\circ} 21^{\prime} 36^{\prime \prime} \mathrm{N}\right.$ y $\left.78^{\circ} 22^{\prime} 39^{\prime \prime} \mathrm{W}\right)$. En cada ocasion se realizaron perfiles de temperatura y conductividad (sonda WTW, LF 196) y se establecio la transparencia con un disco de Secchi (Hydro-Bios 443590). Tambien se tomaron muestras de agua a diferentes profundidades (3, $6,9,14,20,25$, y $29.5 \mathrm{~m}$ ) con una botella muestreadora de dos litros de capacidad (HydroBios 436-132) para analisis de nutrientes, determinando in situ el pH (sonda WTW, 530) y el oxigeno disuelto (sonda WTW, 320).

En el año 1998 se instal6 un sistema de registro automatico de temperatura (multiLogger $\mathrm{mL}$ de 8 canales con sensores tipo Pt 100, ecoTech ${ }_{\circledast}$ ) en una zona cercana a la orilla del lago, donde la profundidad es de $28 \mathrm{~m}$. Con este equipo se registraron tanto la temperatura del ambiente como a seis diferentes profundidades $(0.5,1.0,3.0,9.0,15.0 \mathrm{y}$ $20 \mathrm{~m}$ ). Durante todo el período de muestreo se tomaron datos climatologicos con una estacion de registro automatico $\left(\operatorname{ecoTech}_{\circledast}\right)$.

\section{Análisis de nutrientes}

Una fracción de cada una de las muestras tomadas fue filtrada (filtros de acetato de celulosa, $0,45 \mu \mathrm{m}$ tipo GF/F Whatman) y junto con las muestras sin filtrar heron mantenidas en refrigeración a $4{ }^{\circ} \mathrm{C}$ hasta su evaluación. Los analisis quimicos fueron realizados en la Subsecretaria de Saneamiento Ambiental en Quito (Ecuador), siguiendo los procedimientos dados por la American Public Health Association (1989). Se determinaron los valores de fosforo reactivo soluble (SRP, muestras filtradas, metodo del acido ascorbico), fosforo total (PT, muestras no filtradas, metodo del acido ascorbico previa digestion con acido nítrico y sulfúrico), nitrógeno inorgánico disuelto (DIN, muestras filtradas; para nitrato se utilizó el metodo de reduccion con cadmio y diazotizacion; para nitrito, diazotizacion; para amonio, Nesslirizacion) y nitrogeno organico (muestras no filtradas, metodo Micro- Kjeldahl). Los valores de nitrogeno total (NT) se obtuvieron de la suma de los valores de nitrogeno inorgánico disuelto y nitrogeno organico.

\section{Aspectos biologicos}

Tanto para el analisis del fitoplancton como para la determinación de clorofila $a(\mathrm{Chl} . a)$ las mues- 
tras fueron tomadas a las profundidades arriba mencionadas, con muestras adicionales a $0.5,11$ y $16 \mathrm{~m}$. Las muestras para determinación de fitoplancton fueron fijadas con solución de Lugol (Schwoerbel, 1986) y llevadas a Berlin (Alemania) para su analisis. Estas muestras fueron separadas en tres grupos de acuerdo a la profundidad a la cual fueron tomadas (zona superficial: 0.5 a $9 \mathrm{~m}$; zona media: 11 a $16 \mathrm{~m}$; zona profunda: 20 a $29.5 \mathrm{~m}$ ) y se elaboraron muestras integradas tomando alicuotas de $30 \mathrm{ml}$. Estas muestras se examinaron de acuerdo a la metodologia propuesta por Lund et al. (1958). Para ello se utilizo un microscopio invertido (Zeizz, IM 35) y camaras de sedimentación de 10 y $25 \mathrm{ml}$ de capacidad tipo Utermohl (HydroBios), donde las muestras se dejaron sedimentar durante 24 horas. En cada caso se observaron tantos campos como fueron necesarios para el conteo de por 10 menos 100 ejemplares de cada especie, determinando el area barrida. Relacionando esta ultima con la superficie y el volumen de la camara, se calculo la abundancia de cada especie en ind $/ \mathrm{ml}$. Para la estimación del biovolumen se determinaron las dimensiones geometricas de cada especie $(\mathrm{n}=20)$, y la forma de cada una de ellas se represent6 por un cuerpo geométrico cuyo volumen se calculo a continuacion (Rott, 1981b).

Para la valoracion de Chl. a las muestras fueron filtradas (filtros de fibra de vidrio, Schleicher \& Schuell GF 52) con bombas ma nuales dentro de las 12 horas siguientes al muestreo, registrando en cada caso el volumen filtrado, el cual siempre fue superior a 1.51 . Los filtros fueron marcados y preservados a $-20^{\circ} \mathrm{C}$ hasta el momento de la extraccion y cuantificación, para lo cual se siguio la metodologia propuesta por Nusch (1980). En este método la extraccion se realiza con etanol $(90 \%)$ a $80{ }^{\circ} \mathrm{C} \mathrm{y}$ luego se lleva a cabo una doble lectura espectrofotometrica de las muestras (muestras acidificadas y sin acidificar), para determinar tanto la clorofila $a$ como la feofitina $a$.

Para el estudio del zooplancton se realizaron capturas verticales $(0-10 \mathrm{~m}, 10-20 \mathrm{~m}, 20-30 \mathrm{~m}$ aprox.) con una red para capturas cuantitativas (Hydro-Bios 438040, apertura de entrada $17 \mathrm{~cm}$ de diametro, poro de red de $55 \mu \mathrm{m})$. El volumen filtrado se calculo para cada captura, teniendo en cuenta la longitud de arrastre, la cual se midió en cada caso. Las muestras de zooplancton fueron

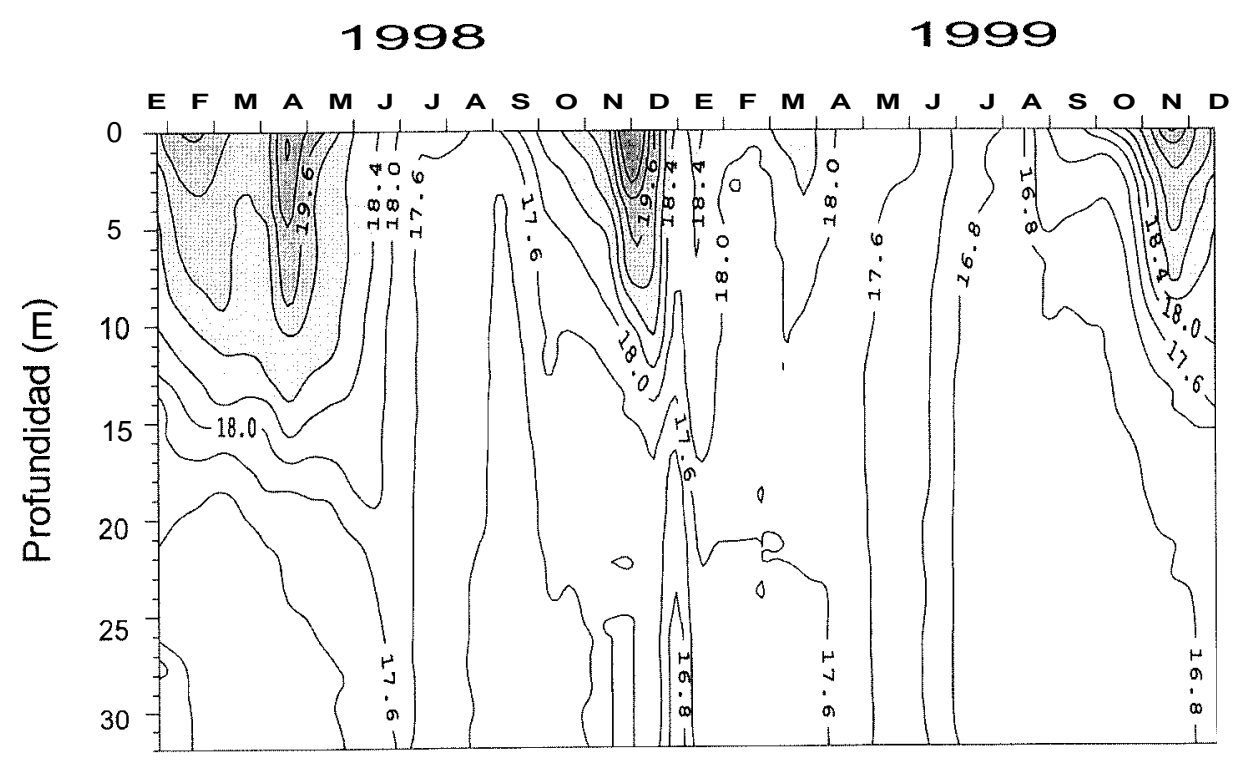

Figura 1. Isopletas de temperatura ("C) del agua en el Lago San Pablo durante 1998 y 1999. Water temperature isophlets ( $\left.{ }^{\circ} \mathrm{C}\right)$ in Luke Sun Pablo (1998-1999). 
fijadas a continuación con solución Haney \& Hall (1973). Tanto las deterrninaciones taxonomicas como las correspondientes determinaciones de abundancia de organismos presentes fueron rea lizadas en la Universidad Técnica de Berlin. Esta valoracion se realizo igualmente con microscopio invertido y camaras volumétricas, siguiendo la metodologia propuesta por McCauley (1984) según la cual la densidad del zooplacton puede ser estimada -dentro de limites estadisticos- por conteo total de varias submuestras tomadas con una pipeta volumetrica (tipo Hansen-Stempel, $1 \mathrm{ml}$ de capacidad, Hidro-Bios 435093). En los casos en que por simple observación la cantidad de zooplancton en la muestra original era baja, se realizo un conteo total de la muestra.

Para la captura de las especies icticas presentes en el lago se contó con la colaboracion de los pobladores de sus riveras.

\section{RESULTADOS}

\section{Parametros fisico-quimicos}

\section{Transparencia}

Los valores de transparencia varian entre $2.1 \mathrm{y}$ $4.2 \mathrm{~m}$, con un valor medio de $3.0 \mathrm{~m}$. Las mediciones máximas se registraron durante los periodos de estratificacion, con valores bajos durante los periodos de mezcla.

\section{Rkgimen tkrmico}

En la figura 1 se presentan las isotermas de la columna de agua durante los aiios 1998 y 1999. Tal como se aprecia, se presenta un período de mezcla hacia mediados del aiio, con periodos de estratificacion entre Septiembre y Mayo aproximadamente. Durante los dos años de muestreo se determino una diferencia maxima de temperatura de apenas 4.5 " $\mathrm{C}$ entre la temperatura minima (16.5 "C) y máxima (21 "C) de la columna de agua. La maxima diferencia dentro de un mismo muestreo fue de 4.1 "C.

Las fases de estratificacion y mezcla presentan variaciones tanto en el momento de su inicio

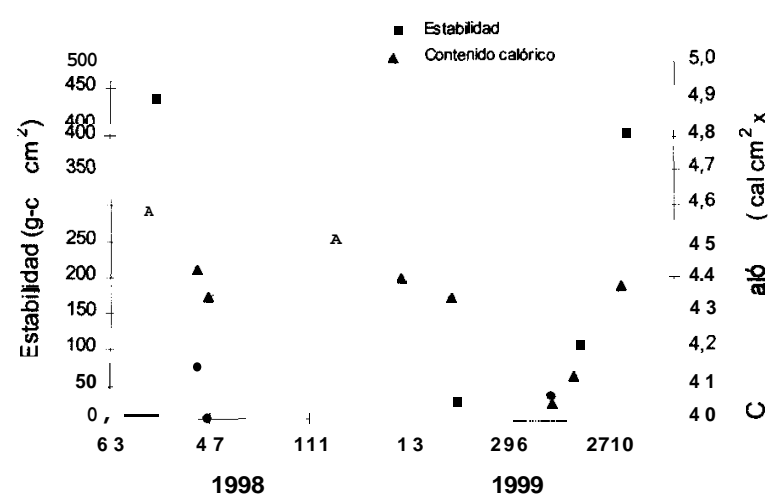

Figura 2. Estabilidad de la estratificacion y contenido de calor en el lago San Pablo durante dias representativos de la estratificacion y de la mezcla. Stratification stability and amount of heat in Lake San Pablo during representative days of the stratification and mixing periods.

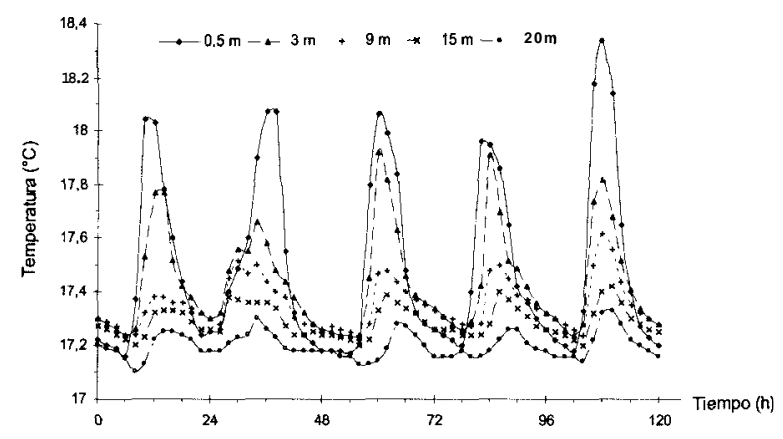

Figura 3. Temperaturas registradas (cada dos horas) a cinco profundidades entre el 15 y el 19 de Junio de 1999, durante la temporada de estratificacion. Se observa la inversion termica que ocurre durante la noche. Temperature changes between 15 and 19 June, 1999, at five depths during stratification. Temperature was recorded every two hours. Thermal inversion at night was apparent.

como en su duración, de acuerdo a las condiciones climáticas imperantes en cada aiio. Es asi como el aiio 1998 inicia con una estratificacion termica que va hasta finales de Junio, dando paso a un período de mezcla desde principios de Julio hasta finales de Agosto. A partir de Septiembre se inicia nuevamente el proceso de estratificacion, el cual se prolonga hasta Abril del aiio 1999. A finales de este mes comienza ya un nuevo período de mezcla, el cual va hasta fines de Agosto. 
1998

1999

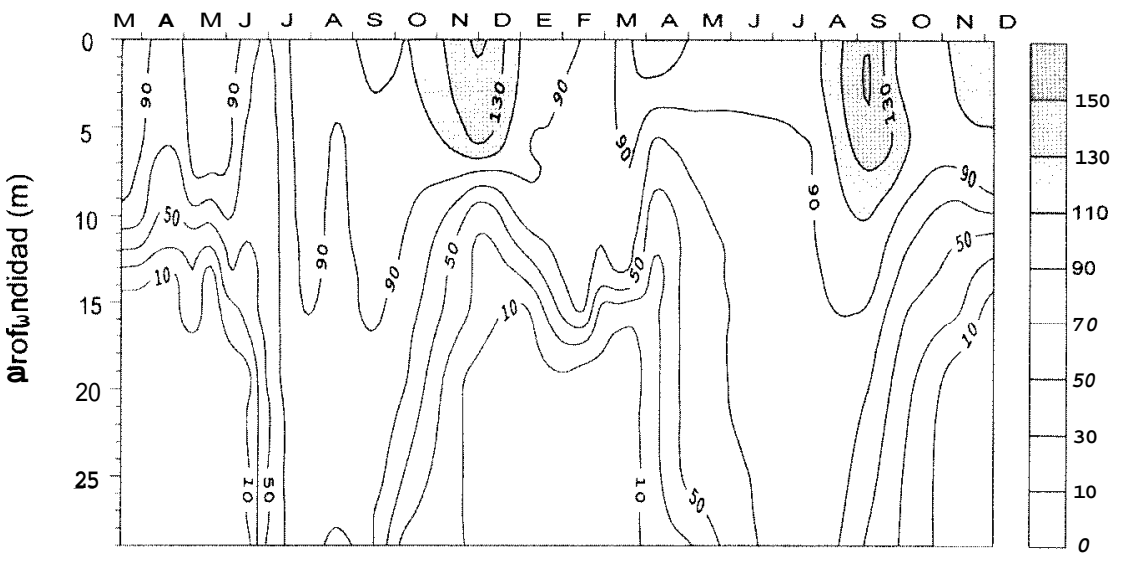

Figura 4. Isopletas de oxigeno disuelto en el Lago San Pablo durante 1998 y 1999. Los valores están dados como porcentaje de saturación de oxigeno. La saturacion se alcanza a una concentracion de $6.4 \mathrm{mgl}^{-1}$ a $20{ }^{\circ} \mathrm{C}$. Isophlets of oxygen percent from saturation in Lake San Pablo (1998-1999). Saturation is reached at a concentration of dissolved oxygen of $6.4 \mathrm{mg} \mathrm{l}^{-1}$ at $20^{\circ} \mathrm{C}$.

Table 1. Valores de concentración de clorofila $a(\mathrm{Chl} a)$, fosforo reactivo soluble (SRP), fosforo total (TP), nitrógeno inorgánico disuelto (DIN), nitrógeno total (TN), asi como valores de la relación entre nutrientes. El valor medio se calculó a partir de los valores encontrados a diferentes profundidades durante dos años de muestreo. Los otros valores corresponden al mínimo y máximo registrado durante este mismo periodo. Chlorophyll a, soluble reactive phosphorus (SRP), total phosphorus (TP) and total nitrogen (TN) concentrations, and relationships between nutrients. Means are calculatedfrom values at different depths over the twoyears of sampling. Other values plotted are the minimum and maximum value found for each variable.

\begin{tabular}{lccccccc}
\hline & Chl. $\mathbf{a}(\boldsymbol{\mu g} / \mathbf{l})$ & $\mathbf{S R P}(\mathbf{m g} / \mathbf{l})$ & TP(mg/l) & DIN(mg/l) & NT (mg/l) & DIN:SRP & TN:TP \\
\hline Promedio & 9.36 & 0.12 & 0.22 & 0.33 & 1.11 & 2.71 & 5.68 \\
Máximo & 32.28 & 0.29 & 0.63 & 1.19 & 3.2 & 20.29 & 16.32 \\
Mínimo & 0.1 & 0.01 & 0.064 & 0.002 & 0.27 & 0.03 & 0.52 \\
\hline
\end{tabular}

1998

1999

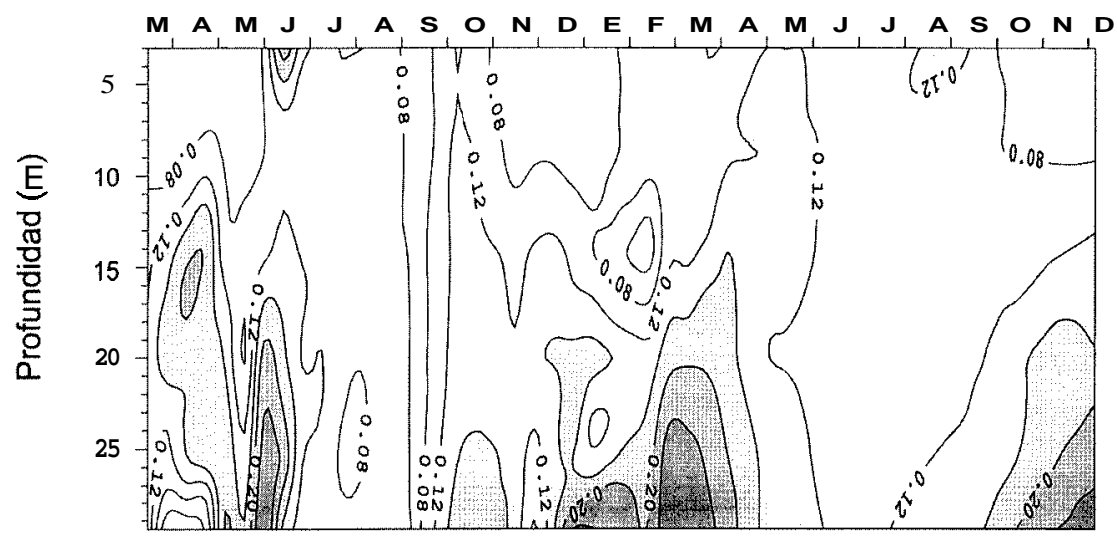

Figura 5. Distribución vertical de fosforo reactivo soluble (SRP, $\mathrm{mgl}^{-1}$ ) durante el tiempo de estudio. Verticaldistribution ofsoluble reactive phosphorus concentrations (SRP, in $\mathrm{mgl}^{-1}$ ) during the study period. 
Durante este ultimo aiio el periodo de circulacion fue mas prolongado, pudiéndose observar una disminucion en la temperatura de la columna de agua desde 17.7" C -registrada en Abril- a 16.7" C alcanzada a finales de Agosto.

Con base en los perfiles de temperatura obtenidos durante los dos aiios de registros y la batimetria del lago, se calcularon los valores correspondientes a la estabilidad de la estratificación (Idso, 1973) y el contenido de calor (Scott, 1964) para algunos dias representativos tanto de la estratificacion como de la mezcla (Fig. 2). La mayor estabilidad se alcanza durante los periodos de maxima estratificacion, cuando el contenido de calor presenta igualmente sus valores mas altos. Con la llegada de la epoca seca y los fuertes vientos vespertinos se inicia el periodo de mezcla, el cual esta asociado directamente con la disminucion de la estabilidad. El muestreo del 2 de Julio de 1998 coincide con un momento de absoluta isotermia, con una temperatura de $17.7^{\circ} \mathrm{C}$ a lo largo de la columna de agua, llegando a anularse totalmente la estabilidad. En este momento se alcanza tambien el valor mas bajo en el contenido de calor para este año. Para el año siguiente, si bien el patron de comportamiento de estas dos variables es similar, hay un cambio en su magnitud. En el aiio 1999 no se detecto una isotermia absoluta durante la circulacion, y la diferencia de temperatura en la columna de agua durante este mismo periodo llego a ser de maximo medio grado. Si bien la estabilidad es baja, no llega a anularse, siendo el contenido calorico menor que el encontrado un aiio atras. Como se anoto antes, el periodo de circulacion durante el aiio 1999 fue mas extenso, debido a que la epoca de vientos se prolongó y la velocidad de estos fue mayor (datos sin publicar). Este ultimo hecho incide en el contenido calorico del lago y determina que la temperatura durante la mezcla este un grado centígrado por debajo de la del aiio 1998.

En la figura 3 se representan las temperaturas registradas a cinco diferentes profundidades entre el 15 y el 19 de Junio de 1999, dias comprendidos dentro de la temporada de circulacion. Este diagrama se realizo a partir de los registros con- tinuos de temperatura, con valores tomados cada dos horas. Como se puede observar, se presentan estratificaciones diurnas e inversiones termicas nocturnas con variaciones en intensidad y duracion dependiendo de la radiación solar, de la velocidad y duracion de los vientos, de la nubosidad y del descenso nocturno de la temperatura ambiente.

\section{Quimica del agua}

Las aguas del lago presentaron valores de $\mathrm{pH}$ entre 7.6 y 9.0, con los valores mas altos hacia las capas superficiales y tendencia a la homogeneidad durante la circulacion.

La conductividad mostro uniformidad a 10 largo del aiio, con valores entre 280 y $337 \mu \mathrm{S} / \mathrm{cm}$, siendo estos caracteristicos de lagos ecuatoriales y de montaiia donde la mineralización es deficiente (Payne, 1986; Donato, 199I).

La estratificacion y la mezcla del cuerpo de agua determinan tambien el comportamiento de algunos parametros quimicos, afectando asi la recirculacion y distribucion de nutrientes y el contenido y distribucion de oxigeno. En la figura 4 estan representadas las isopletas de oxigeno disuelto como porcentaje de saturación. La saturation en las aguas del lago es alcanzada a una concentracion de $6.4 \mathrm{mg} / \mathrm{l}$ de oxigeno a $20^{\circ} \mathrm{C}$. Se observa que la distribucion de oxigeno sigue el mismo patron observado para la temperatura, con valores de $70 \%$ durante la mezcla y de sobresa turacion en los estratos superiores durante la etapa de estratificacion. Durante esta última se presenta un deficit efectivo de oxigeno en las aguas profundas, hecho que esta relacionado con el incremento en la concentracion de fosforo reactivo soluble (SRP).

Los valores medios, maximos y minimos de Chl. $a$, de las concentraciones de nitrógeno y fosforo, y de las relaciones entre estos nutrientes se presentan en la Tabla 1. En 10 referente al SRP, se determino una alta concentracion con los valores mas bajos en las capas superficiales $(0.05-0.12 \mathrm{mg} / \mathrm{l}$ ) y concentraciones mayores en la zona profunda $(<0.3 \mathrm{mg} / \mathrm{l} \mathrm{P})$. Como se aprecia en la figura 5, la mayor concentracion de SRP se detecto durante las épocas de estratificacion, cuan- 


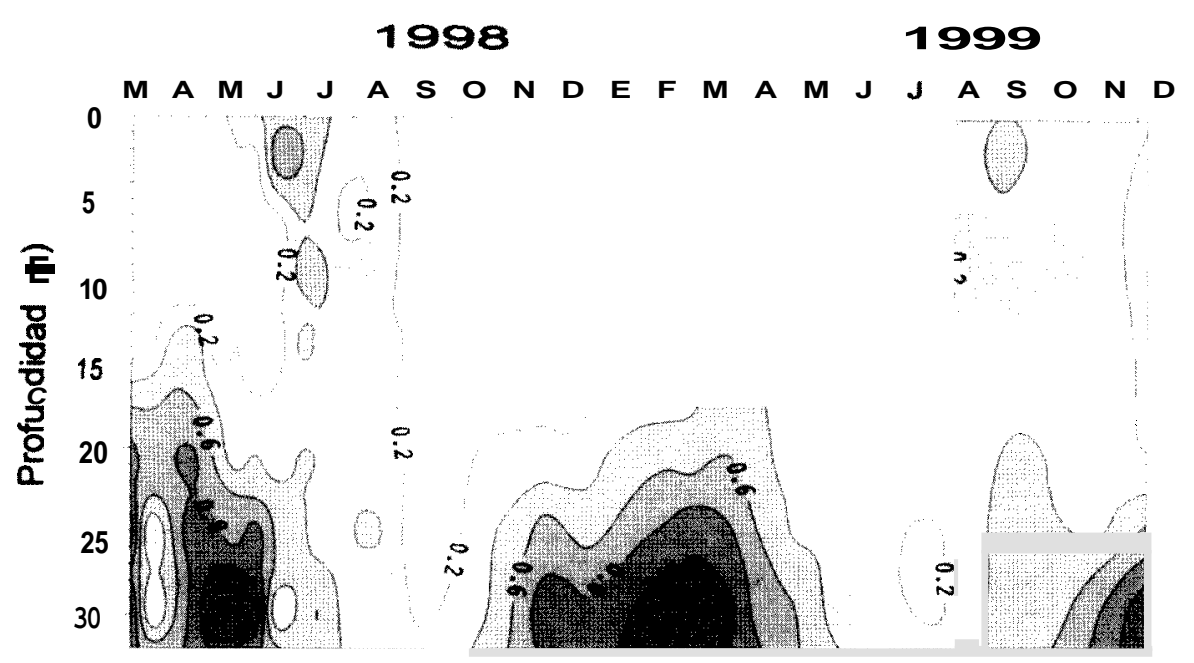

Figura 6. Distribución vertical de nitrogeno inorganico disuelto (DIN, mgl-1) durante el tiempo de estudio. Verticaldistribution of dissolved inorganic nitrogen (DIN, in mgl-1) during the study period.

do se presentan condiciones anoxicas en los sedimentos, lo cual favorece la liberación de fosforo (Bostrom et al., 1982; Bostrom et al., 1988). La concentración de fosforo total en el zona fotica llega normalmente a estar por encima de $0.2 \mathrm{mg} / \mathrm{l}$ y solamente en muy pocas ocasiones alcanza valores mayores de $0.6 \mathrm{mg} / 1$.

Las concentraciones de nitrogeno inorganico disuelto representadas en la figura 6 son bajas y, de acuerdo a estudios realizados para las zonas temperadas, caracteristicas de lagos oligo-mesotroficos (Vollenweider, 1986). Estas concentraciones presentaron sus picos maximos en la zona trofolitica y los valores minimos en la zona trofogenica durante las epocas de estratificacion. Es importante anotar que el amonio es la forma quimica de mayor peso dentro de esta fracción. En terminos generales, este representa mas del $90 \%$ durante la estratificacion, pudiendo llegar a menos del $20 \%$ durante la mezcla.

Las concentraciones de nitrogeno total son elevadas y se encuentran entre 0.27 y $3.2 \mathrm{mg} / 1$, con un promedio de $1.11 \mathrm{mg} / \mathrm{l}$. No obstante, estos niveles se pueden considerar de baja magnitud con respecto a los niveles de fosforo, ya que la relacion NT:PT (en peso) se encuentra durante la mayor parte del ciclo hidrologico por debajo de
10, lo que indicaria una limitación potencial de la productividad a causa del nitrogeno. Esta relacion esta influenciada por la presencia de las fracciones organicas, las cuales no estan disponibles biologicamente, por 10 cual se ha calculado la relacion DIN:SRP, que proporciona información útil y puntual sobre las deficiencias de nutrientes, ya que la productividad en un momento dado puede estar marcada por las especies quimicas en solución (Payne, 1986; Wurtsbauch, et. al.,1992). En promedio se determino un relacion DIN:SRP de 2.7, con valores muy bajos en la zona fotica $(0.18$ como valor medio hasta $9 \mathrm{~m}$ de profundidad) y algo mas altos hacia los estratos profundos (1.27, entre 14 y 30 metros), 10 cual confirmaría una limitacion a causa del nitrogeno.

\section{Parametros biologicos}

\section{Fitoplancton}

Las especies fitoplanctonicas encontradas, asi como su permanencia y abundancia, se presentan en la Tabla 2. El numero de especies es bajo para un sistema de las caracteristicas del lago San Pablo, aunque hay que señalar que otros autores 
Table 2. Presencia y abundancia de las especies fitoplanctonicas encontradas. El indice de presencia se establecio en base al porcentaje de muestreos en los cuales se encontro la especie: r=rara (0-25\%), e=esporádica $(25-50 \%), \mathrm{f}=$ frecuente $(50-75 \%), \mathrm{p}=$ permanente $(75-100 \%)$. El indice de abundancia se establecio en base al numero de organismos de cada especie encontrado durante los dos años de estudio con respecto al total de organismos: $\mathrm{mb}=$ muy baja, $\mathrm{b}=\mathrm{baja}, \mathrm{m}=$ media, $\mathrm{a}=\mathrm{alta}$. Presence/absence list and abundances ofphytoplankton species recorded The presence/absence index is calculatedfrom the percentage of sampling occasions when the given species wasfound. $r$ is rare 10 -25\% of sampling occasions); $e$ is sporadic (25-50\%) f-frequent (50-75\%) andp-permanent (75-100\%). The abundance index was calculated as the number of individuals of each species over total abundance. mb is very low; $b$-low; $m$ - medium abundance and $a$ is high abundance.

Especie

\begin{tabular}{|c|c|}
\hline Presencia & Abundancia \\
\hline $\mathrm{e}$ & $\mathrm{b}$ \\
\hline $\mathbf{f}$ & $\mathrm{b}$ \\
\hline $\mathrm{r}$ & $\mathrm{b}$ \\
\hline $\mathrm{r}$ & $\mathrm{mb}$ \\
\hline $\mathrm{r}$ & $\mathrm{mb}$ \\
\hline$f$ & $\mathrm{a}$ \\
\hline $\mathrm{P}$ & $\mathrm{a}$ \\
\hline $\mathrm{f}$ & $\mathrm{b}$ \\
\hline $\mathrm{P}$ & $\mathrm{m}$ \\
\hline $\mathrm{e}$ & $\mathrm{b}$ \\
\hline e & $\mathrm{b}$ \\
\hline f & $\mathrm{b}$ \\
\hline $\mathrm{e}$ & $\mathrm{b}$ \\
\hline $\mathrm{P}$ & $\mathrm{b}$ \\
\hline $\mathrm{e}$ & $\mathrm{b}$ \\
\hline $\mathrm{r}$ & $\mathrm{mb}$ \\
\hline $\mathrm{r}$ & $\mathrm{mb}$ \\
\hline $\mathrm{r}$ & $\mathrm{mb}$ \\
\hline $\mathrm{r}$ & $\mathrm{b}$ \\
\hline $\mathrm{r}$ & $\mathrm{b}$ \\
\hline $\mathrm{r}$ & $\mathrm{mb}$ \\
\hline $\mathrm{r}$ & $\mathrm{mb}$ \\
\hline$P$ & $\mathrm{a}$ \\
\hline $\mathrm{r}$ & $\mathrm{mb}$ \\
\hline $\mathrm{r}$ & $\mathrm{mb}$ \\
\hline $\mathrm{r}$ & $\mathrm{mb}$ \\
\hline $\mathrm{r}$ & $\mathrm{mb}$ \\
\hline
\end{tabular}

han reportado especies que en el presente estudio no han sido encontradas (Rott, 1981a; Steinitz-Kannan et al. 1983). La comunidad esta dominada por la Clorofita Scenedesmus linearis, así como por la Euglenofita Trachelomonas volvocina y la diatomea filamentosa Aulacoseira granulata, especies con alta abundancia relativa y permanencia estable en el lago. Otras especies que se encuentran durante casi todo el año pero en menor abundancia son Pediastrum boryanum y Neglectella sp. La presencia de cianobacterias es esporadica e igualmente de baja abundancia. Unicamente entre Julio y Diciembre del año 1998 se encontro Microcystis aeruginosa.

El numero de celulas determinado para la zona trofogenica ( $0.5-9 \mathrm{~m}$ de profundidad) esta entre 
1998

1999

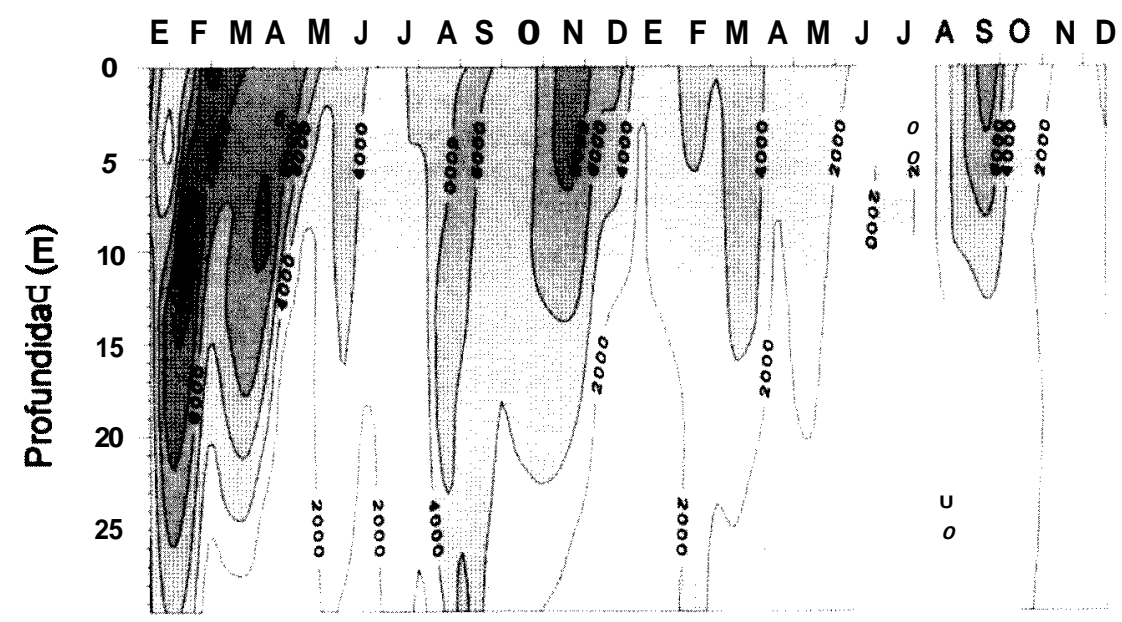

Figura 7. Distribución de abundancia fitoplanctonica $\left(\right.$ ind $\left.^{-1}\right)$ durante el tiempo de estudio. Verticaldistribution of phytoplankton abundance (cells $l^{-1}$ ) during the study period.

1000 y $12000 \mathrm{ind} / \mathrm{ml}$ y para la zona trofolitica (11 - $29.5 \mathrm{~m}$ de profundidad) entre $200 \mathrm{y}$ $7500 \mathrm{ind} / \mathrm{ml}$. En la figura $7 \mathrm{se}$ muestran estos valores en forma de isolineas de igual concentracion. La mayor abundancia se encontro durante la estratificacion térmica en los primeros meses del afio 1998, cuando la transparencia en la columna de agua es mayor. Durante el período de mezcla se presentan los valores mas bajos. Este patron se repite durante el afio 1999 pero con valores menores. Las concentraciones estan marcadas fundamentalmente por la alta abundancia de $S$. linearis, aunque tambien son de importancia $P$. boryanum, $A$. granulata y $T$. volvocina. El biovolumen se encuentra entre $70 \times 10^{3}$ y $6000 \times 10^{3} \mu \mathrm{m}^{3} / \mathrm{ml}$ (Fig. 8). Contrario a 10

1998

ロ999

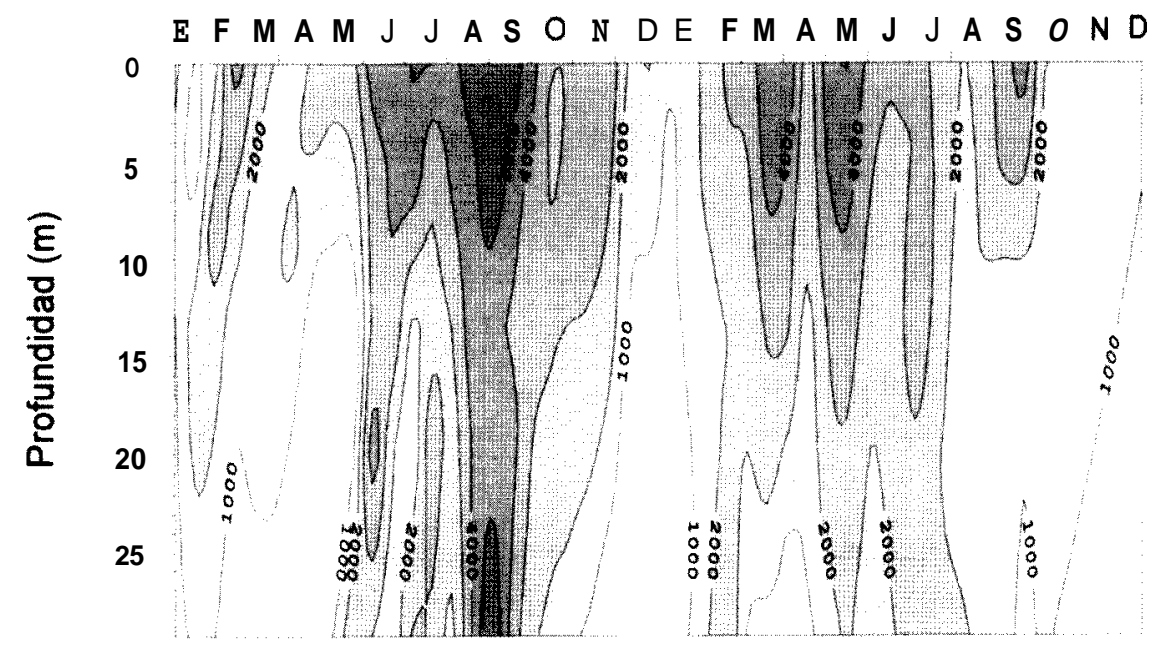

Figura 8. Isopletas de biovolumen de fitoplancton $\left(\mu \mathrm{m}^{3} \mathrm{ml}^{-1} \mathbf{x} 10\right)$. Phytoplunkton biovolume isophlets $\left(\mu \mathrm{m}^{3} \mathrm{ml}^{-1} \mathbf{x} 10\right)$ 
Table 3. Especies zooplanctonicas encontradas en el lago San Pablo. Zooplankton species recorded in Lake San Pablo from January 1998 to December 1999.

\begin{tabular}{ll}
\hline Cladocera & Daphnia pulicaria \\
Copepoda & Metacyclops mendocinus \\
Rotifera & Anuraeopsisfissa \\
& Asplachna sp. \\
Euchlanis sp. \\
Keratella cochlearis \\
Keratella tropica \\
Lecane bulla \\
Lecane luna \\
Polyarthra sp. \\
Pompholyx complanata \\
Trichocerca similis
\end{tabular}

descrito para la abundancia, los valores máximos se detectaron durante y despues de los periodos de mezcla. Estos datos se confirman con los obtenidos para la Chl. $a$, la cual alcanza los valores mas altos durante esta misma fase (maxima concentración determinada: $32.8 \mu \mathrm{g} / \mathrm{l}$ a finales de Junio de 1999), con concentraciones menores durante los periodos de estratificacion (Fig. 9). Esta aparente contradicción entre la abundancia y el biovolumen esta explicada por la presencia de Trachelomonas volvocina durante la mezcla, cuyo volumen celular puede llegar a ser 10 veces mayor al de los otros representantes del fitoplancton.

\section{Zooplancton}

En la tabla 3 se relacionan las especies zooplanctonicas encontradas. Esta comunidad es extremadamente pobre, constituida por poblaciones poco abundantes de especies cosmopolitas. En el lago se encuentran solamente dos especies de crustaceos: Daphnia pulicaria y Metacyclops mendocinus, un ciclopoideo carnívoro. Los rotiferos estan representados por diez especies, de las cuales solo cinco llegan a ser permanentes y moderadamente abundantes: Anuraeopsis fissa, Keratella tropica, Polyarthra vulgaris, Trichocerca similis y Asplanchna sp. En la figura 10 se muestran los valores de abundancia determinados para el año 1998, donde los rotiferos se presentan como la suma de las 5 especies mas abundantes. Este grupo represent6 el $75.9 \%$ de la abundancia total de organismos, mostrando la mayor variación en los valores de abundancia debido sobre todo al incremento en la poblacion de A.fissa durante Septiembre.

Daphnia pulicaria fue encontrada durante todo el aiio, con una abundancia maxima de $12 \mathrm{ind} / \mathrm{l}$ en

1998 1999

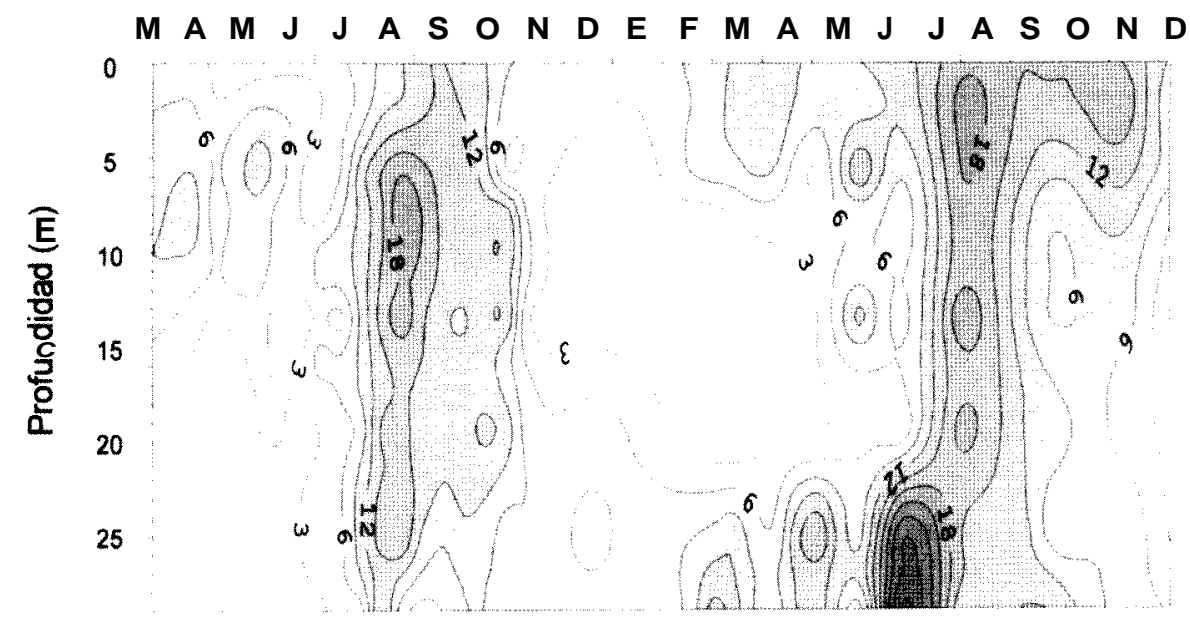

Figura 9. Distribución vertical de clorofila $a\left(\mu \mathrm{gl}^{-1}\right)$ durante el tiempo de estudio. Verticaldistribution of chlorophyll a $\left(\mu \mathrm{gl}^{-1}\right)$ during the study period. 


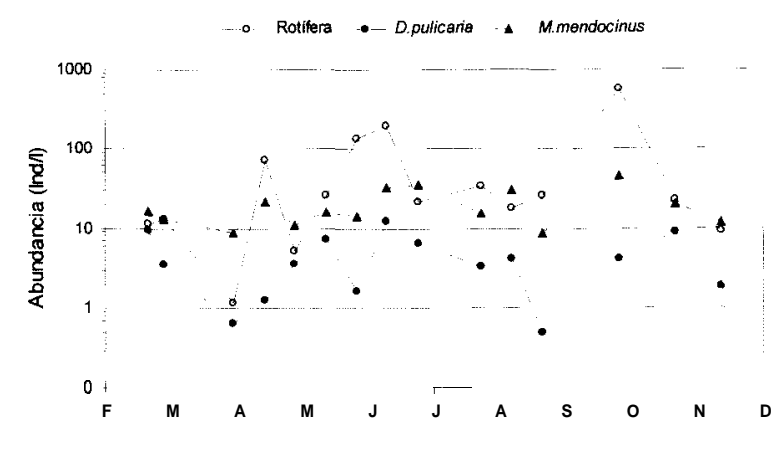

Figura 10. Abundancia del zooplancton durante el año 1998. Los rotiferos estan representados como la suman de las cinco especies mas frecuentes. M. mendocinus corresponde a las suma de copepoditos avanzados y adultos. Zooplankton abundance during 1998. Rotifers plotted are the sum of the five most frequent species. $M$ mendocinus are advanced copepodites plus adults.

Julio y minima de 0.5 ind.// en Septiembre. Su abundancia relativa fue de $4.5 \%$.

Metacyclops mendocinus es una especie de amplia distribución en America Central y del Sur (Loffler, 1963; Reid, 1985; Valdivia, 1988; Reid et. al., 1990). Tambien se le encuentra en las Islas Azores y en algunas regiones de Europa (Defaye \& Dussart, 1991). Los valores representados en la figura 10 corresponden a la suma de adultos y de copepoditos avanzados, ya que son estos estadios los que se consideran carnívoros. La abundancia durante 1998 estuvo entre 9 y 44 ind/l., con una abundancia relativa del $19.5 \%$. Los valores maximos se encontraron durante y despues del periodo de circulacion. A pesar de que la variación anual en la abundancia de $M$ mendocinus no es muy marcada, se observa una correspondencia entre esta y las variaciones encontradas en los rotiferos y en $D$. pulicaria, lo cual puede indicar la interaccion depredador-presa.

\section{Peces}

Las especies encontrados fueron identificadas como Micropterus salmoides y Carassius carassius x C. auratus, introducidas hace ya varias décadas. Estas especies son capturadas esporádicamente y en bajo numero, correspondiendo la mayoria a $A 4$ salmoides. Los pobladores de la zona reportan la presencia en décadas pasadas de la trucha arco iris (Oncorhynchus mykiss), especie introducida en varios paises andinos, la cual al parecer ha sido desplazada totalmente por $M$. salmoides, ya que no se observo durante el tiempo de estudio y tampoco existen reportes actuales sobre su presencia.

\section{DISCUSIÓN}

El comportamiento termico que se presenta en el lago San Pablo a 10 largo del ciclo hidrologico esta caracterizado por un periodo de estratificacion que coincide con las temporada de Iluvia, y un periodo de mezcla durante la epoca seca. La estratificacion esta marcada por una baja diferencia de temperaturas a 10 largo de la columna de agua, por lo cual se puede considerar debil.

El inicio de la epoca seca hacia mediados del año -el llamado verano- y la consecuente aparicion de fuertes vientos vespertinos suministra la energia necesaria para romper la estratificacion generada durante el invierno y dar inicio a un periodo de circulacion de las masas de agua. En este proceso la intensidad y la duracion de los vientos es decisiva en el balance energetico, determinando la duracion del periodo de mezcla y la temperatura de la columna de agua durante esta epoca. Otro hecho relevante es la estratificacion diaria que es generada durante las horas de mayor radiación. Al amainar los vientos hacia las 18:00 horas, es el descenso de la temperatura ambiental el que ocasiona pérdidas de calor en las capas superficiales de la columna de agua, causando incluso inversiones termicas. Estos bruscos cambios de temperatura pueden generar corrientes de convección, produciendo mezclas nocturnas que pueden llegar hasta los $20 \mathrm{~m}$ de profundidad. Este fenomeno fue tambien observado durante las epocas de estratificacion, pero no se presenta todas las noches, ya que depende de las condiciones climaticas imperantes cada dia (Gunkel \& Casallas, 2001).

De acuerdo a todo 10 anterior, el lago San Pablo podria ser clasificado como monomictico calido, ya que presenta un solo periodo de mezcla a lo largo del año con temperaturas por encima de 
$16^{\circ} \mathrm{C}$. La formacion de una estratificacion diaria durante gran parte del aiio indicaria a su vez un regimen de tipo polimictico, lo cual se considera caracteristico de los lagos de alta montaiia (Lewis, 1987; Roldan, 1992). Por la altura a la cual se encuentra el lago y las caracteristicas encontradas, este representaria un tipo intermedio o de transición entre los lagos calidos tropicales de zonas bajas y los lagos de alta montaña o paramo, demostrando la complejidad del regimen termico en estos sistemas. A este respecto Lewis (2000) hace una referencia sobre el sistema de clasificación, indicando la confusion que existe sobre la tipologia de los lagos tropicales basada en las descripciones de Hutchinson y Lofler (1956), a la cual considera basicamente incorrecta cuando se aplica en las regiones tropicales. El mismo autor afirma que los lagos tropicales son fundamentalmente monomicticos, con variaciones hacia la polimixis o la meromixis dependiendo de su profundidad. Como anota SteinitzKannan et al. (1983), la generalización sobre la polimixis de los lagos altos de las zonas tropicales de Africa y America es ampliamente aceptada, a pesar de que no es totalmente satisfactoria cuando es aplicada a los lagos ecuatorianos, ya que todos se estratifican de vez en cuando y la mayoria pueden mezclarse como consecuencia de lluvias y vientos, especialmente durante la noche, tal como se comprobo en el lago San Pablo.

En los procesos descritos, tanto la morfometria del lago como el clima regional -y en especial el regimen de vientos- juegan un papel preponderante. Los valores de estabilidad $\mathbf{y}$ de contenido de calor demuestran como dichos elementos son decisivos en el balance energetico, ya que determinan las ganancias, las transferencias y las pérdidas de calor, 10 que al final establece el ritmo de circulacion y mezcla. Otro aspecto climatico de gran influencia en la definición de patrones estacionales es el regimen de lluvias, el cual determina incrementos en el aporte de aguas con material suspendido y nutrientes disueltos. Esta dependencia ha sido observada en lagos cálidos tropicales (Melack, 1979; Payne, 1986; Masundire, 1994) y altoandinos (Donato, 1991;
Mora \& Tellez, 1991), donde la maxima productividad coincide con la epoca de lluvias y la minima con el período seco, temporadas estas que corresponden a la circulacion y a la estratificación respectivamente. En el caso del lago San Pablo, si bien se mantiene el patron de productividad durante y despues de la circulacion, este período no corresponde con el regimen de lluvias. Esto significa que los aportes externos generados durante esta estacion tienen otra dinamica, pudiendo ser en parte utilizados por los productores primarios y en parte acumulados en los sedimentos hasta la llegada del verano. Tal como anota Nilssen (1984), el viento es el factor determinante en la circulacion de muchos tipos de lagos tropicales, teniendo mayor influencia en este proceso que las fluctuaciones estacionales de la radiación y de la temperatura atmosferica.

Como se pudo constatar en el presente estudio, las fases de estratificacion y de circulacion determinan a su vez la distribución y la concentración de las especies quimicas en el agua (SRP, DIN) $y$ el suministro de oxigeno hacia las capas infe riores. La interacción de estos procesos determina las temporadas y la magnitud en que se presentan los mayores valores de biomasa fitoplanctónica, estimados mediante el biovolumen y la Chl. a.El desarrollo de la biomasa esta marcado asi por los periodos de mezcla durante la epoca de fuertes vientos, cuando hay una oxigenacion de las capas profundas y los nutrientes acumulados durante la estratificacion son puestos nuevamente a disposición de los productores primarios, alcanzandose durante y despues de estas temporadas los picos de Chl. a. Sin embargo, y de acuerdo a las concentraciones de nutrientes encontradas, se puede afirmar que el desarrollo del fitoplancton es bajo. Es un hecho que la relacion NT:PT establece durante gran parte del aiio una limitacion potencial debida al nitrogeno, siendo esta limitacion mas evidente cuando se considera la relacion DIN:SRP. Esta limitacion por nitrogeno se ha encontrado ya en algunos lagos altoandinos (Wurtsbauch et al., 1985; Payne, 1986; Donato, 1991; Roldan, 1992). Esta circunstancia estableceria una ventaja competitiva para las cianobacterias fijadoras de nitrogeno, 
con 10 que se esperaria un aumento considerable en su abundancia, hecho que sin embargo no se presenta. El bajo desarrollo de las cianobacterias en sistemas deficientes en nitrogeno ha sido ya reportado en algunos lagos tropicales (Henry et al., 1978; Richerson et al., 1986; Lewis \& Weibezahn, 1987) y esta relacionado con la alta intensidad luminica, la cual inhibe la fijacion de nitrógeno atmosferico (Fogg, 1971).

Los bajos valores de abundancia fitoplanctonica encontrados en San Pablo, asi como el bajo numero de especies presentes, pueden estar influenciados por varios factores, entre los cuales se puede mencionar el efecto de la alta intensidad luminica (Richerson et al., 1992), la radiación ultravioleta (Löffler, 1964; Hader, 1995; Hessen et al., 1997; Kinzie, 1998; Hader, 1999), la herbivoria (Carpenter et al., 1993; Cruz-Pizarro et al., 1994) y la posible perdida de biomasa hacia las zonas profundas (Lewis, 1974; Knoechel \& Kalff, 1975; Imberger, 1985; Gunkel \& Casallas, 2001). La predominancia en el lago San Pablo de formas de vida propias de sistemas turbulentos (Margalef, 1978) observada ya en otros lagos altoandinos (Iltis, 1992; Donato,1991; Mora \& Tellez, 1991), asi como los altos valores de Chl. $a$ encontrados a $29.5 \mathrm{~m}$, pueden ser un indicio de la validez de esta ultima hipotesis. Todos estos aspectos tendrán que ser analizados en futuras investigaciones para establecer su efecto sobre la comunidad.

Establecer las relaciones tróficas entre productores y consumidores esta mas alla de las metas del presente trabajo; sin embargo, se pueden hacer algunas observaciones a este respecto. A la luz de los resultados obtenidos, parece no existir un patron definido en la relacion productores y consumidores. Es sabido que el fitoplancton constituye la base alimenticia del zooplancton herbivoro, si bien aquel no siempre cubre las necesidades nutricionales de este (Gonzales de Infante, 1988). En muchos sistemas se ha establecido una correlación directa entre las variaciones estacionales de estas dos comunidades, influenciada por la entrada de nutrientes durante el invierno (Infante, 1982; Payne, 1986, Cisneros \& Mangas, 1991). No obstante, tambien se han encontrado casos donde florecimientos fitoplanc- tónicos tienen un efecto negativo sobre el desarrollo de especies de Daphnia (Gasiunaite \& Olenina, 1998). Si se considera la baja abundancia de D. pulicaria y la variación observada durante 1998, no se puede definir una tendencia que pueda relacionarse con el desarrollo del fitoplancton. A partir de Marzo se observa un descenso en su abundancia (Fig. 10), a pesar de los altos valores de biomasa fitoplanctonica. $\mathbf{S i}$ bien se aprecia un ligero aumento al inicio del verano, a este le sigue un descenso hasta alcanzar el valor mas bajo en Agosto, cuando los valores de Chl. $a$ son mas elevados. Cruz-Pizarro, et al. (1994) encontraron en un lago oligotrofico de alta montaiia, donde D. pulicaria constituye el $98 \%$ de la biomasa heterotrofica y su abundancia es comparable a la encontrada en San Pablo, que la oferta alimenticia no suple las necesidades nutricionales de esta especie. De manera analoga, el bajo desarrollo del fitoplancton en el lago San Pablo, asi como el efecto de la predación por parte de $M$. mendocinus, podrian estar limitando el desarrollo de D. pulicaria. Es de anotar que la presencia de estas dos especies de crustáceos, relativamente grandes, puede ser un indicativo de la ausencia o de la baja presencia de depredadores pelagicos de mayor tamaño. Según se observó, las especies de peces presentes ocupan preferencialmente las zonas litorales donde la oferta alimentaria es mayor.

En 10 referente a los rotiferos, se observo un aumento en su abundancia durante el período seco, 10 que seguramente tiene que ver con un aumento en el material suspendido debido a la circulación de la columna de agua. En los lagos tropicales se ha observado que los rotiferos planctonicos pueden encontrarse en cualquier ambiente sin presentar una relacion precisa con el estado trofico (Esteves, 1988). En su desarrollo no solo la disponibilidad de alimento es importante, ya que las asociaciones de rotiferos caracteristicas de los trópicos estan ampliamente determinadas por la temperatura y la salinidad, al igual que por la altitud $\mathbf{y}$ el tamaño de los cuerpos de agua (Green, 1994).

Por ultimo, el estimar el estado trofico actual del lago con base en las variables analizadas y a 
partir de los modelos existentes resulta algo complejo, ya que conforme a las pautas establecidas por la OECD (1982) y el CEPIS (1990), los valores de Chl. $a$ indicarian un estado mesotrófico, a la vez que las concentraciones de nitrógeno total encontradas clasificarian al lago como moderadamente eutrofico. No obstante, los altos niveles de fosforo total indicarian un estado altamente eutrofico. Es evidente que la deforestacion, la actividad agricola, los procesos de erosion y la falta de tratamiento de aguas residuales han producido alteraciones en el equilibrio del sistema, afectando la productividad y las cadenas troficas, llevando al lago a un proceso de eutroficacion.

Por las caracteristicas expuestas, los sistemas acuaticos tropicales de montaiia no pueden ser explicados con los madelos que se han desarrollados para los lagos tropicales de zonas bajas (CEPIS, 1990) ni con aquellos desarrollados para los lagos de zonas temperadas (Vollenweider, 1968; Lewis, 1987; Baigun \& Marinone, 1995). Ya que los lagos y embalses tropicales presentan una mayor susceptibilidad a la eutroficacion (Lewis, 2000), es evidente la necesidad de ampliar los conocimientos sobre estos sistemas para definir modelos propios que permitan hacer diagnosticos acerca de la evolucion de los mismos, con el fin de tomar medidas apropiadas de mitigación y control.

\section{AGRADECIMIENTOS}

Esta investigación ha sido financiada por la Asociacion de Investigación Alemana (Deutsche Forschungsgemeinschaft, DFG) y el Ministerio Alemán de Cooperación Tecnica y Desarrollo (Bundesministerium fur wirtschaftliche Zusammenarbeit und Entwicklung, BMZ), a quienes agradecemos por su apoyo.

Agradecemos igualmente al Departamento de Medio Ambiente (Facultad de Ingenieria Civil) de la Escuela Politécnica Nacional del Ecuador, por su colaboracion en el trabajo de campo.

\section{BIBLIOGRAFÍA}

APHA. 1989. Standard Methods for the Examination of Water and Wastewater. American Public Health Association, Washington, D.C. $17^{\text {th }}$ Ed. 1550 pp.

BAIGUN, C. \& M. MARINONE. C.1995. Cold-temperate lakes of South America: Do they fit northern hemisphere models? Archiv Hydrobiol., 135: 23-51.

BOSTRÖM, B., M. JANSSON \& C. FORSBERG. 1982. Phosphorus release from lake sediments. Arch. Hydrobiol. Beih. Ergebn. Limnol., 18: 5-59.

BOSTROM, B., J. M. ANDERSEN, S. FLEISCHER \& M. JANSSON. 1988. Exchange of phosphorus across the sediment-water interface. Hydrobiologia, 170: 229-244.

CARPENTER, S. R., J. A. MORRICE, J. J. ELSER, A. S. AMAND \& N. A. MACKAY. 1993. Phytoplankton community dynamics. En: The trophic cascade in lakes. Carpenter, S. R. \& Kitchell, J. F. (eds.): 116-152. Cambridge University Press, Cambridge.

CEPIS. 1990. Metodologías simplificadas para la evaluacidn de eutroficación en lagos cálidos tropicales. Centro Panamericano de Ingenieria Sanitaria y Ciencias del Ambiente. Organización mundial de la Salud. 39 pp.

CISNEROS, R. O. \& I. MANGAS. 1991. Zooplankton studies in a tropical lake (Lake Xolotlán, Nicaragua). Verh. Internat. Verein. Limnol., 24: 1167-1170.

CRUZ-PIZARRO, L.; I. RECHE \& P. CARRILLO. 1994. Plankton dynamics in a high mountain lake (Las Yeguas, Sierra Nevada, Spain). Indirect evidence of ciliates as food source for zooplankton. Hydrobiologia, 274: 29-35.

DEFAYE, D. \& B. DUSSART. 1991. Sur les Crustaces Copépodes des Acores. Annls Limnol., 287: $119-132$.

DONATO. J. C. 1991. Fitoplancton y aspectos físicos y quimicos de la laguna de Chingaza (Una laguna tropical de alta montaiia). Cuad. Divulg., 11:1-18.

DUCHARME, A. 1975. Estudio fisicoquímico $y$ bioldgico del lago de Tota. Revision bibliografica de la Empresa de Acueducto y Alcantarillado de Bogota.

ESTEVES, F. A. 1988. Fundamentos de Limnologia. Editora Interciencia Ltda., Rio de Janeiro.

FOGG, G. E. 1971. Nitrogen fixation in lakes. Plant and Soil. Special vol.:393-401

GALARRAGA, E.; O. ZEVALLOS, P. TOLEDO, C. CRIOLLO, N. ROBELLY \& J. RUALES. 1992. Estudio para la Recuperacidn y Proteccidn de la 
Zona Turistica de los Lagos de la Provincia de Imbabura con fines de Equilibrio Ecologico y Promoción Turistica. Ministerio de Relaciones Exteriores, República del Ecuador.

GASIUNAITE, Z. R. \& I. OLENINA. 1998. Zooplankton-phytoplanktoninteractions: a possible explanation of the seasonal succession in the Kursiu Marios lagoon. Hydrobiol., 363: 333-339.

GONZALEZ DE INFANTE, A. 1988. El Plancton de las Aguas Continentales. Secretaria General de la Organización de Estados Americanos. Programa Regional de Desarrollo Cientifico y Tecnologico. Washington, D.C. 130 pp.

GREEN, J. 1994. The temperate-tropical gradient of planktonic Protozoa and Rotifera. Hydrobiologia, 272: 13-26.

GUNKEL, G. 2000. Limnology of an Ecuatorial Mountain Lake in Ecuador, Lago San Pablo. Limnologica, 30: 113-120.

GUNKEL, G. \& J. CASALLAS. 2001. Limnnology of an Ecuatorial High Mountain Lake, Lago San Pablo, Ecuador: Limitation of Lake Productivity by Deep Diurnal Mixing. Proceedings of the international Association of Theoretical and Applied Limnology. (en prensa).

HADER, D. 1995. Influence of ultraviolet radiation on phytoplankton ecosystems. In: Algae, Environment and Human Affairs. Wiessner, W., Schnepf, E. \& Starr, R.C. (eds.): 41-55. Biopress Ltd., Bristol.

HADER, D. 1999. Effects of solar UV-B radiation on aquatic ecosystems. Verhandlungen der Gesellschaft für Okologie, 29: 463-471.

HANEY, J. F. \& D. J. HALL. 1973. A preservation technique for Cladocera. Limnol. Oceanogr: 18: 331-333.

HENRY, R., E. M. PELLEGRINI CARAMASHI \& J. G. TUNDUSI. 1978. Survey of ecological factors in a shallow tropical reservoir. Rev. Bras. Biol., 38: 171-175.

HESSEN, D. O., H. J. DE LANGE \& E. VAN DONK. 1997. UV- induced changes in phytoplankton cells and its effects on grazers. Freshwat. Biol., 38: 513524.

HOLDRIGE, L. 1978. Ecología basada en las zonus de vida. Instituto Interamericano de Ciencias Agricolas. San Jose, Costa Rica.

HUTCHINSON, G. E. \& H. LOFLER. 1956. The thermal classification of lakes. Pro. Nat. Acad. Sci., 42: $84-86$.

IDSO, S. B. 1973. On the concept of lake stability. Limnol. Oceanogr., 18: 681-683.
ILTS, A. 1992. Lake Titicaca. A Synthesis of Limnological Knowledge. Kluwer Academci Publishers Dordrecht, The Netherlands. 573 pp.

IMBERGER, J. 1985. The diurnal mixed layer. Limnol.Oceanogr., 30: 737-770.

INAMHI, Instituto Nacional de Meteorologia e Hidrologia Centro Nacional de Análisis y Pronostico. Boletines Meteorologicos. Quito, Ecuador.

INFANTE, A. 1982. Annual variation in abundance of zooplankton in Lake Valencia (Venezuela). Arch. Hydrobiol., 93: 194-208.

KIERSCH, B., R. MUHLECK \& G. GUNKEL. 2001. Las macrofitas de algunos lagos altoandinos del Ecuador y su posible utilización como bioindicadores de eutrofización. Revista de Biologia Tropical, (en prensa).

KINZIE III RA, A. A. BANASZAK, M. P. LESSER. 1998. Effects of ultraviolet radiation on primary productivity in a high altitude tropical lake. Hydrobiologia, 385: 23-32.

KNOECHEL, R. \& KALFF, J. 1975. Algal sedimentation: The cause of a Diatom-blue-green succession. Verh. Internat. Verein. Limnol., 19: 745-754.

KOSTE, W. \& K. BOTTGER. 1989. Rotatorien aus Gewassern Ecuadors. Amazoniana, 10 (4): $407-$ 438.

KOSTE, W. \& K. BOTTGER. 1992. Rotatorien aus Gewassern Ecuadors II. Amazoniana, 12 (2): 263303.

LEWIS, W. M. Jr. 1973. The thermal regime of lake Lanao (Philippines) and its theoretical implications for tropical lakes. Limnol. Oceanogr., 18(2): 200217.

LEWIS, W. M. Jr. 1974. Primary production in the plankton community of a tropical lake. Ecological Monographs, 44: 377-409.

LEWIS, W. M. Jr. 1987. Tropical limnology. Ann. Rev. Ecol. Syst., 18: 159-184.

LEWIS, W. M. Jr. 2000. Basis for the protection and management of tropical lakes. Lakes \& Reservoirs. Research and Management, 5: 35-48.

LEWIS, S. M. \& H. F. WEIBEZAHN. 1987. Chemistry, energy flow, and community structure in some Venezuelan fresh-waters. Verein.Limnol., (2/3): 145-207.

LOFFLER, H. 1963. Zur Ostrakoden- und Copepodenfauna Ekuadors. Arch. Hydrobiol., 59: 196-234.

LOFFLER, H. 1964. The limnology of tropical highmountain lakes. Verh. Interant. Verein. Limnol., 15: 176-193. 
LUND, J. W. G., C. KIPLING \& E. D. LE CREN. 1958. The Inverted microscope method of estimating algal numbers and the statistical basis of estimations by counting. Hydrobiologia, 11: 143-170.

McCAULEY, E. 1984 The estimation of the abundance and biomass of zooplankton in samples. En: A manual on methodsfor the assesment of secondaryproductivity infresh water. IBP Hand Book 17. J.A. Downing \& F. Rigler (eds.): 228-265. Blackwell Scientific Publ, London.

MARGALEF, R. 1978. Life forms of phytoplankton as survival alternatives in an unstable environment. Oceanol. Acta, 1 : 493-509,

MASUNDIRE, H. M. 1994. Seasonal trends in zooplankton densities in Sanyati basin, Lake Kariba: multivariate analysis. Hydrobiologia, 272: 211 230.

MELACK, J. M. 1979. Temporal variability of phytoplankton in tropical lakes. Oecologia, 44: 1-7

MELACK, J. M. 1996. Recent developments in tropical limnology. Verh. Internat. Verein. Limnol., 26: 211-217.

MILLER, A., M. KANNAN \& P. A. COLINVAUX. 1984. Limnology and primary productivity of Andean and Amazonian tropical lakes of Ecuador. Verh. Internat. Verein. Limnol., 22: 1264-1270.

MORA M., G. \& L. S. TELLEZ . 1991. Estudio limnológico del lago de Tota (Boyacá - Colombia). Tesís de grado, Biologia. Universidad Nacional de Colombia, Bogotá.105 pp.

NILSSEN, J. P. 1984. Tropical Lakes - Functional ecology and future development: The need for a process oriented approach. Hydrobiologia, 113: 231-242.

NUSCH, E. A. 1980. Comparison of different methods for chlorophyll and phaeopigment determination. Arch. Hydrobiol. Beih. Ergebn. Limniol., 14: 14-36.

OECD. 1982. Eutrophication of waters - monitoring, asssessment and control. OECD Paris, $154 \mathrm{pp}$.

OSBORNE, P. L. 2000. Tropical Ecosystems and Ecological Concepts. $1^{\text {st }}$ ed. Cambridge Universuty Press, Cambridge. 464 pp.

PAREDES CASTILLO, D. 1994. Rehabilitación ambiental y aprovechamiento sustentable de los lagos de San Pablo, Yaguarcochay Cuicocha en la provincia de Imbabura. Consultoria Socio-economica y Planificacion. Quito. Primer borrador. $25 \mathrm{pp}$

PAYNE, A. I 1986. The ecology of tropical lakes and rivers. John Wiley \& Sons, Manchester. 301 pp.

POWELL, T., M. H. KIRKISH, P. J. NEALE, \& P. J. RICHERSON. 1984. The diurnal cycle of stratifi- cation in Lake Titicaca: Eddy diffusion. Verh. Internat. Verein. Limnol., 22: 1237-1243.

REID, J. W. 1985. Chave de identificaçao e lista e referencias bibliograficas para as especies continentales sulamericanas de vida libre da orden Cyclopoida (Crustacea, Copepoda). Bolm. Zool. Univ.S. Paulo, 9: 17-143.

REID, J. W., J. A. MOLINA. \& M. M. FUKUSCHIMA. 1990. Metacyclops leptopus totensis, new subspecies (Crustacea, Copepoda) from Lago de Tota, Colombia. Proc. Biol. Soc. Washington, 103: 674680.

RICHERSON, P. J., P. J. NEALE et al. 1986. Patterns of temporal variation in lake Titicaca. A high altitude tropical lake. I. Background physical and chemical processes and primary production. Hidrobiologia, 138: 205-220.

RICHERSON, P. J. 1992. Lake Titicaca. A Synthesis of Limnological Knowledge.Kluwer Academic Publishers

ROLDAN, G. 1992. Fundamentos de Limnología Neotropical. Editorial Universidad de Antioquia, Medellin. 529 pp.

ROTT, E. 1981a. Ein Beitrag zur Algenflora andiner Hochlandseen Ecuadors (Sudamerika). Ber. nat.med. Ver. Innsbruck, 68: 13-29.

ROTT, E. 1981b. Some results from phytoplankton intercalibrations. Schweiz. Z. Hydrol., 43: 34-62.

SCHWOERBEL, J. 1986. Methoden der Hydrobiologie - Süßwasserbiologie. Gustav Fisher Verlag, Stuttgart. 369 pp.

SCOTT, J. T.1964. A comparison of the heat balance of lakes in winter. Tech. Rep. 13. Dept. of Meteorol., Univ. of Wisconsin, Madison. 138 pp.

STEINITZ-KANNAN, M., P. A. COLINVAUX \& R. KANNAN. 1983. Limnological Studies in Ecuador 1. A Survey of chemical and physical properties of Ecuadorian lakes. Archiv Hydrobiol. Supplement 65: 61-105.

VALDIVIA, R. S. 1988: Lista de Cladoceros dulceacuicolas del Peru. Amazoniana, 10:283-297.

VOLLENWEIDER, R. A. 1968. Scientific Fundamentals of the Eutrophication of Lakes and Flowing Waters, with Particular Reference to Nitrogen and Phosphorus as Factors in Eutrophication. OECD Reporte DASiCSIi68.27. $192 \mathrm{pp}$.

WURTSBAUGH, W. A., W. F. VINCENT, C. L. VINCENT, H. J. CARNEY, P. J. RICHERSON \& R. ALFARO. 1992. Nutrients and nutrient limitation of phytoplankton V.6. En: Lake Titicaca. A 
Synthesis of Limnological Knowledge. Dejoux \& A. Iltis (eds): 147-160. Kluwer Academic Publischers. WURTSBAUCH, W. A.,W. F. VINCENT, R. ALFARO TAPIA, C. L. VINCENT \& P. J. RICH-
ERSON. 1985. Nutrient limitation of algal growth and nitrogen fixation in a tropical alpine lake, Lake Titicaca (Perú/Bolivia). Freshwat. Biol., 15: 185-195. 